\title{
A STUDY ON THE BENEFITS OF TELEOPHTHALMOLOGY IN EYE CARE MANAGEMENT
}

\author{
K. S. Rajiv Krishna ${ }^{1}$ \\ ${ }_{1}^{1}$ Assistant Professor, Department of Ophthalmology, Andhra Medical College/Government Regional Eye Hospital, Visakhapatnam, \\ Andhra Pradesh, India.
}

\begin{tabular}{l}
\hline ABSTRACT \\
\hline BACKGROUND \\
Teleophthalmology allows us to overcome various hurdles and improve quality, access and affordability in eye care management. \\
Logistic problems in utilisation of eye care services, concentration of ophthalmologists and corporate hospitals in metros and big \\
cities and lack of resources for investment as well as inadequate trained manpower is some of the reasons for the high morbidity \\
due to various ophthalmic diseases.
\end{tabular}

The objective of the study is to show the benefits of Teleophthalmology in diagnosing and managing eye diseases.

\section{MATERIALS AND METHODS}

A total of 1200 patients with ophthalmic complaints from different age groups attending two primary health centres linked to the Regional Eye Hospital, Visakhapatnam were studied and analysed over a period of six months, i.e. from 01-06-2017 to 31-12-2017. The study was mainly done by real time video conferencing. The PHCs are connected to the REH, Visakhapatnam via BSNL internet service, a Government of India enterprise.

\section{RESULTS}

In the six-month study, 1200 patients visiting the PHCs were evaluated. Among them 50\% had refractive errors followed by cataract in 35\%. Other diseases involving retina and corneas were seen in $3 \%$ and $1 \%$ of the patients respectively. Post cataract surgery complications like PCO was noticed in $5 \%$ of the patients.

\section{CONCLUSION}

Teleophthalmology is a boon to the ophthalmic patients with rural background and plays a key role in eye care management at the gross root level in developing country like India.

\section{KEY WORDS}

Teleophthalmology, Primary Health Centres, Eye Care Management.

HOW TO CITE THIS ARTICLE: Krishna KSR. A study on the benefits of teleophthalmology in eye care management. J. Evolution Med. Dent. Sci. 2018;7(28):3229-3233, DOI: $10.14260 /$ jemds/2018/726

\section{BACKGROUND}

Teleophthalmology is the integration of electronic information and medical technology, by which people in remote and underserved areas can get access to specialised expert eye care, especially in subspecialties of ophthalmology like cornea, retina, adnexal and orbital diseases 1,2 . Teleophthalmology is a rapidly developing field, thanks to application of ophthalmic science by telephone, the internet or other networks for the purpose of consultations and on occasion carrying out examinations or diagnostic procedures. $^{3}$ The integration of the technologies enabled simultaneous transmission of voice, video and biometrics data representing an important development incentive. The continuous improvement and growth of communication technology reached the internet era, producing an inexpensive global network of widespread technology. 4 In addition the possibility to store extensive image, audio and text information with the ability to provide services has been explored more, enabling robust advances in teleophthalmology in a short period.

In comprehensive ophthalmology, image-based triage has been observed to reduce face-to-face appointments by $16 \%$

'Financial or Other Competing Interest': None.

Submission 29-05-2018, Peer Review 25-06-2018,

Acceptance 01-07-2018, Published 09-07-2018.

Corresponding Author:

Dr. K. S. Rajiv Krishna,

\#2-45-14/1, GF-101, Veitla Park View Apartment, MVP Colony,

Sector-11, Visakhapatnam-530017, Andhra Pradesh, India.

E-mail: rajivkrishna9@yahoo.in

DOI: $10.14260 / \mathrm{jemds} / 2018 / 726$ to $48 \%$ avoiding unnecessary appointments, shortening waiting lists and improving the coordination of specialist services. In addition, teleophthalmology has been considered comparably effective or superior in providing accurate diagnosis when compared to face-to-face consultation ${ }^{5}$ in diagnosing chronic blurred vision causes. Regarding satisfaction with remote consultation, due to the recent advances in information technology and as a cost and timesaving modality teleophthalmology showed high acceptance regardless of patient's age. Health care accessibility is considered the cornerstone of teleophthalmology. This objective would be accomplished by decreasing travel distance and in turn care delay, enabling tertiary and primary care independent of the patient's location. Patient care delivered in local health facilities promotes cost reduction 6 by diminishing the need for transportation to tertiary care centres or for specialists to travel for health care delivery. Moreover, electronic consultations have been shown to cost less than face-to-face consultations, representing significant savings. Comprehensive patient information available at the evaluation also may minimise the need for exam or tests replication, reducing extra cost and time to establish diagnosis and management, and avoiding unnecessary referrals ${ }^{7}$ representing a time-effective advantage. As in other medical fields, telemedicine has been introduced in ophthalmology care and has been applied and absorbed differently by the diverse subspecialties as an ascending trend. 


\section{Objective of the Study}

The above study is focused on evaluating and analysing various ophthalmic diseases, which can be addressed through teleophthalmology, thereby reflecting the benefits of teleophthalmology in eye care management at tertiary level in the Regional Eye Hospital, Visakhapatnam, Andhra Pradesh.

\section{MATERIALS AND METHODS}

We performed retrospective descriptive study. All the patients who attended the eye clinics at primary health centres connected via teleophthalmology during the study period were registered. A predesigned proforma was used to collect the data and analysed using MS Excel software.

Two primary health centres Yelamanchili and Paderu were connected to Regional Eye Hospital, Visakhapatnam, Andhra Pradesh via BSNL internet service. The population residing in the Primary Health Centre (PHC) area and those dwelling in neighbouring PHC areas were made aware of the teleophthalmology setup. 1200 patients from different age groups were included in the study over a period of six months from 1-6-2017 to 31-12-2017. Clinical details of all the patients were noted for analysis. The patients were treated daily from Monday to Saturday by real time video conferencing. Paramedical ophthalmic officer recorded all the patient identification details. Main complaint, duration, clinical history, present, past, medical, family, treatment and personal history were also taken. Visual acuity was recorded using standard Snellen chart. Anterior segment examination was done by slit lamp examination. Posterior segment examination was done using direct ophthalmoscopy. The teleophthalmology unit at REH was manned by Assistant Professors with support from Senior Residents and Postgraduates. Specialist consultations were taken as needed. If any abnormality was noted, the patient was immediately referred to the main hospital, REH. Depending upon the case, necessary investigations were ordered. Treatment for minor ailments was given on live video conferencing. Health education on ocular hygiene, proper nutrition, need to undergo eye surgeries wherever indicated was provided. Counselling for proper usage of spectacles, especially in the young ametropes was given. Postoperative follow-ups and routine follow-ups of other diseases were taken up.

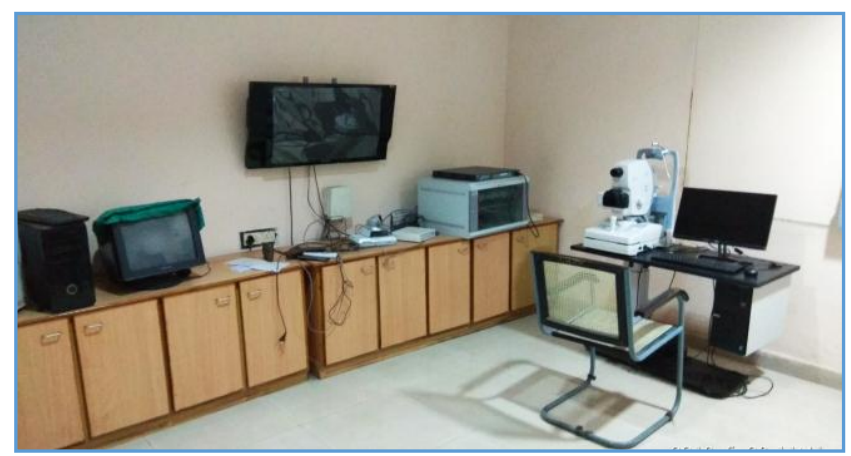

Figure 1. Teleophthalmology Setup at Regional Eye Hospital, Visakhapatnam

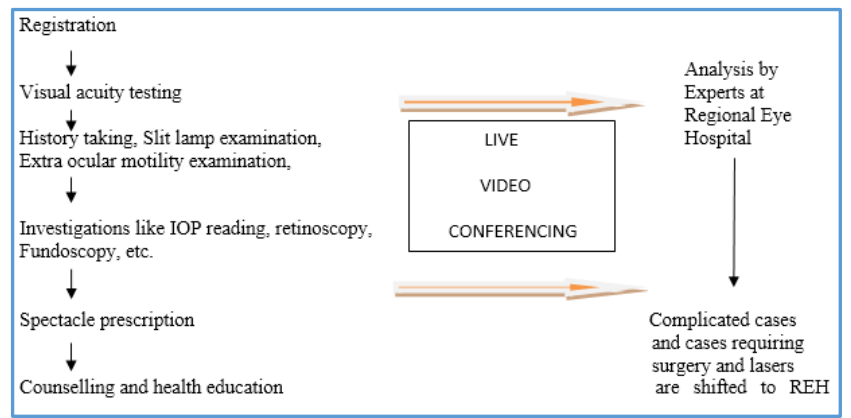

Figure 2. Flow Chart showing the Working Pattern in our Setup

Indications for teleconsultation ${ }^{8}$ were refractive errors, unexplained visual loss, glaucoma suspects and glaucoma cases, all patients with diabetes and hypertension, strabismus cases, infections of the conjunctiva or cornea, retinal pathologies, cases where vision did not improve with pin hole and best possible refraction other than cataract, and cause of visual loss was undetermined, any history of previous ocular surgeries associated with visual impairment, any case where either the optometrist had a doubt or if the patient wished to interact with the Consultant at REH.

\section{Inclusion Criteria}

All the patients attending the teleophthalmology clinic during the study period of six months were included in the study.

\section{RESULTS}

In the 1200 patients studied, prevalence of various ophthalmic diseases was analysed. Refractive errors contributed to $50 \%$, cataracts $35 \%$, pterygium and diabetic retinopathy $3 \%$, glaucoma, squint and corneal diseases contributed 1\%. Orbital disorders and neurophthalmology cases were prevalent in $0.5 \%$ of the study group. Posterior capsular opacification, one of the most common complication after cataract surgery was noted in $5 \%$ of the patients.

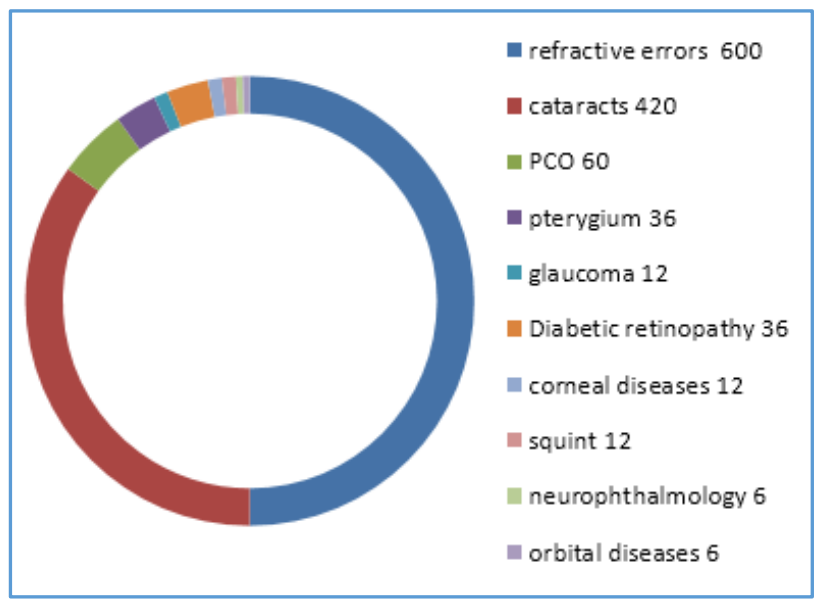

\section{Graph 1. Showing the Prevalence of various} Ophthalmic Diseases in the Study Group

Male patients $52 \%$ and female patients $48 \%$ contributed to the study. $26 \%$ of the patients were in paediatric age group, $34 \%$ were adults and $40 \%$ were from geriatric age group. Among the patients attending the teleophthalmology clinics, $72 \%$ of the patients were from nearby areas and $28 \%$ 
were from far off places. 95\% of the patients attending the clinics were satisfied with the care and treatment and 5\% expressed some grievances. Majority of the patients required medical $32 \%$ and/or surgical $40 \%$ treatment. $20 \%$ of the patients were given counselling on various ophthalmic diseases, nutrition deficiencies and management, proper usage of spectacles etc. $8 \%$ of the patients underwent laser procedures.

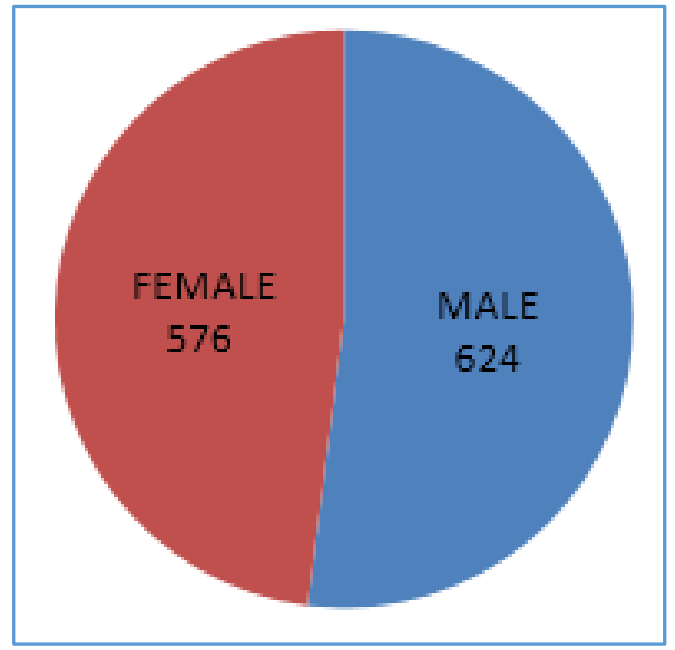

Graph 2. Showing Male-to-Female Population in the Study

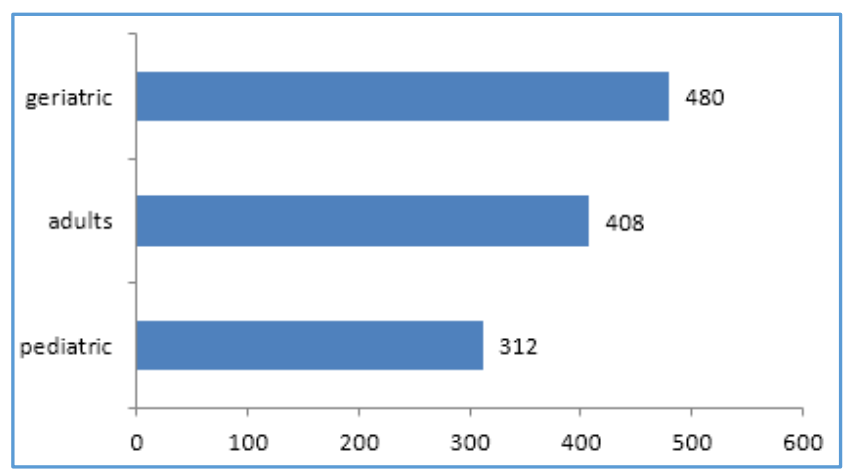

Graph 3. Showing Children, Adults and Elderly Patients in the Study

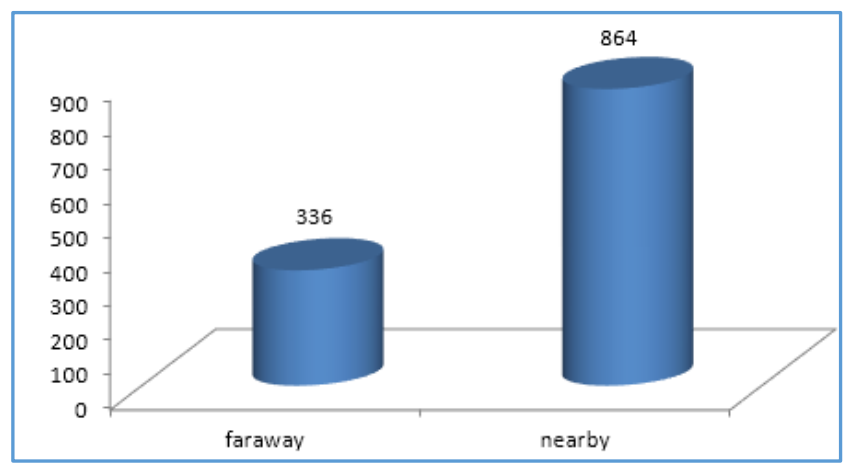

Graph 4. Showing People attending from far away and nearby places to the Clinic

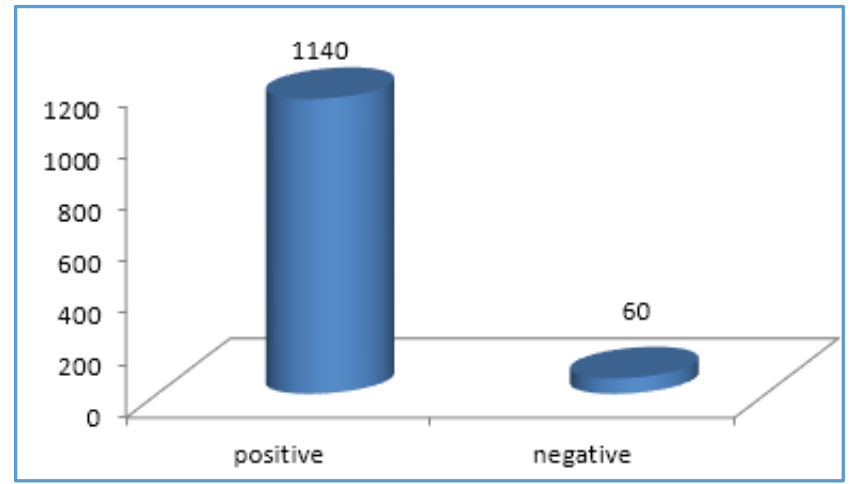

Graph 5. Showing People giving Positive Feedback for Teleophthalmology Clinics

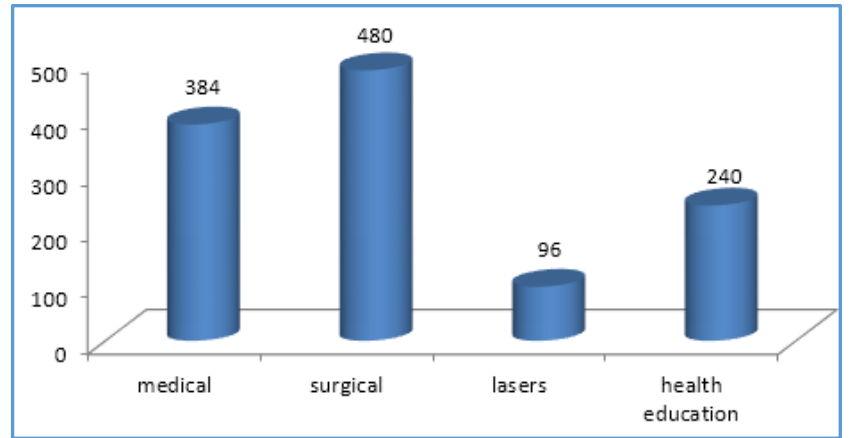

Graph 6. Showing various Eye Care Treatment Modalities given at REH for patients attending Teleophthalmology Clinic

\section{DISCUSSION}

Teleophthalmology, a ramification from telemedicine initially consisted of descriptive studies and only by the end of the 1990s did teleophthalmology application begin to be assessed through analytic studies. ${ }^{9}$ Teleophthalmology might be one of the most challenging applications of telemedicine, given its level of detailed elements and need for refined imaging. Financial planning is very essential for installing and maintenance of teleophthalmology units, as it may require sophisticated and multiple equipments just like other branches of medicine. 10 In addition to telecommunication features such as high image resolution, audio clarity, good signal reception and pre-specified protocols are required to provide ophthalmic clinical data. ${ }^{11}$ Over a period of time, teleophthalmology evolved from various limitations and attained the present stage incorporating the rapid advances in the field of communications and technology. Even though limitations remain regarding the availability of telecommunication equipment, teleophthalmology has attained a state where it is giving dependable and reproducible results in eye care management.

The benefits of teleophthalmology 12 are diagnosis and formulation of treatment plans for diabetic retinopathy, visual acuity testing and refraction, low vision consultations, cataract screening, glaucoma screening, anterior segment imaging, continuing medical education by tele-education, transferring of ultrasound, electro-oculography and electroretinography images, employment facilities to the local unemployed youths and outreach of quality services to remote rural and underserved areas where specialised medical care is not available. 
This study at our institute showed the benefits of teleophthalmology in diagnosing and management of common eye diseases prevalent in the community. Majority of the common conditions diagnosed could be addressed through teleophthalmology barring a few. Refractive errors and cataracts had the major share among the eye diseases. John et al in their study also obtained similar results. ${ }^{8}$ Retinoscopy was done whenever required and suitable glasses were prescribed. Cataract cases were identified, necessary preliminary investigations done and if found within normal limits were referred to the Regional Eye Hospital for surgeries. Late post-operative follow-ups were done. Complications like posterior capsular opacification were noted and were referred for YAG capsulotomy. Patients with increased intraocular pressures and patients with hypertension and diabetes were properly counselled and referred as needed. Other diseases with less prevalence were dealt appropriately.

\section{CONCLUSION}

Teleophthalmology helps in screening for common, but vision-threatening diseases that have the potential to pose major public health problems. From a population point of view, teleophthalmology greatly reduces travel time and capital, 13 thereby facilitating utilisation of eye care services even in the remotest of villages where such services are not freely available. This in turn greatly helps in the eye care management of drown-trodden people in rural India. Major causes of preventive blindness like refractive errors diagnosed in the clinics can easily be corrected by prescribing spectacles, and cataracts can be managed by timely surgery. Teleophthalmology also provides some employment opportunities to the rural youth. Patient satisfaction ${ }^{14}$ is also very much positive in the clinics, which is a very vital factor in the success of teleophthalmology. There are few studies available on this subject and I hope in the coming future more research and studies will be conducted. Kambiz Bahaadinbeigy ${ }^{15}$ in his research on publications pertaining to studies on teleophthalmology concluded that majority of the studies, 95\% gave a positive opinion on teleophthalmology. The limitations ${ }^{16}$ in teleophthalmology are high installation costs, requirement of high definition imaging which mandates sophisticated equipment and state of art technology with dependable power supply and backup.

In conclusion, this study proves that delivery of comprehensive eye care to the rural population in Visakhapatnam district of Andhra Pradesh through teleophthalmology is a viable, successful programme. With comprehensive eye examination, we can prevent many common causes of avoidable blindness. If teleophthalmology services can be extended to remote areas through nongovernmental and charity organisations, we might be able to provide reasonable solution towards reducing the prevalence of avoidable blindness in a developing country like India. In addition to eye care delivery, the potential for distance learning, continued education and clinical trial collaboration are some applications of teleophthalmology that should be explored in greater detail in the future.

\section{ACKNOWLEDGEMENTS}

I sincerely thank all faculty of the Institute, my Senior Residents and Postgraduate students for their help. Last but not the least my gratitude to the patients with whom we personally interacted via live video conferencing.

\section{REFERENCES}

[1] Verma M, Raman R, Mohan RE. Application of teleophthalmology in remote diagnosis and management of adnexal and orbital diseases. Indian J Ophthalmology 2009;57(5):381-4.

[2] Boucher MC, Desroches G, Garcia-Salinas R, et al. Teleophthalmology screening for diabetic retinopathy through mobile imaging units within Canada. Can J Ophthalmology 2008;43(6):658-68.

[3] Sharma LK, Rajput M. Telemedicine: socio-ethical considerations in the Indian milieu. The Medico-Legal Journal 2009;77(Pt 2):61-5.

[4] Yogesan K, Constable IJ, Eikelboom RH, et al. Teleophthalmic screening using digital imaging devices. Aust N Z J Ophthalmo 1998;26 Suppl 1:S9-S11.

[5] Liddy C, Rowan MS, Afkham A, et al. Building access to specialist care through e-consultation. Open Med 2013;7(1):e1-e8.

[6] Kirsh S, Carey E, Aron DC, et al. Impact of a national specialty e-consultation implementation project on access. Am J Manag Care 2015;21(12):e648-54.

[7] Caffery LJ, Farjian M, Smith AC. Telehealth interventions for reducing waiting lists and waiting times for specialist outpatient services: a scoping review. J Telemed Telecare 2016;22(8):504-12.

[8] John S, Sengupta S, Reddy SJ, et al. The Sankara Nethralaya mobile teleophthalmology model for comprehensive eye care delivery in rural India. Telemedicine Journal and E-health: the official Journal of the American Telemedicine Association 2012;18(5):382-7.

[9] Flowers CW Jr, Baker RS, Khanna S, et al. Teleophthalmology: rationale, current issues, future directions. Telemed J 1997;3(1):43-52.

[10] Vidmar DA. Plea for standardization in teledermatology: a worm's eye view. Telemed J 1997;3(2):173-8.

[11] Perednia DA, Gaines JA, Butruille TW. Comparison of the clinical informativeness of photographs and digital imaging media with multiple-choice receiver operating characteristic analysis. Arch Dermatol 1995;131(3):292-7.

[12] Prathiba V, Rema M. Teleophthalmology: a model for eye care delivery in rural and underserved areas of India. International Journal of Family Medicine 2011;2011:683267.

[13] Tan IJ, Dobson LP, Bartnik S, et al. Real-time teleophthalmology versus face-to-face consultation: a systematic review. J Telemed Telecare 2017;23(7):629-38.

[14] Host BK, Turner AW, Muir J. Real-time teleophthalmology video consultation: an analysis of patient satisfaction in rural Western Australia. Clin Exp Optom 2018;101(1):129-34. 
[15] Bahaadinbeigy K, Yogesan K. Advances in teleophthalmology: summarising published papers on teleophthalmology projects. March 22nd 2011. DOI: $10.5772 / 13595$.
[16] Grisolia ABD, Abalem MF, Lu Y, et al. Teleophthalmology: Where are we now? Arq Bras Oftalmol 2017;80(6):401-6. 